SIR-I believe that the mechanism proposed by Kirkpatrick and Jenkins ${ }^{2}$ is unlikely to offer a sufficient explanation for the maintenance of diploid sex.

Of critical importance in assessing the potential role of the segregation advantage $^{1}$ in maintaining diploid sex is the fact that the gradual accumulation of beneficial homozygous loci by a sexual population can only lead to gradual increases in its relative fitness. Hence, a significant time period (Kirkpatrick and Jenkins provide an estimate of $\sim 10^{6}$ generations) would be required before the level of homozygosity in a sexual population could counterbalance the supposedly inherent twofold fitness advantage of asexual reproduction. But throughout these many generations the asexual population would enjoy a large, slowly diminishing selective advantage, which should result in the competitive elimination of the sexual population long before its fitness approaches that of the asexual population. In addition, competition with a more fit asexual population would lead to a continuous reduction in the size of the sexual population, slowing its relative rate of production of new beneficial mutant alleles, and further reducing its competitive prospects. Even if the sexual population eliminated the asexual population, new asexual mutants, starting with the same genetic complement as the sexual population, would again enjoy a selective advantage for, say, $\sim 10^{6}$ generations. In the absence of any advantage to sex other than an initially higher rate of fixing homozygous loci, the repeated appearance of such mutants would keep a sexual population at a constant disadvantage.

The segregation advantage cannot explain the evolution of sex or its maintenance in haploid forms ${ }^{1}$. However, the existence of sex in species with predominantly haploid life cycles implies a selective advantage sufficient to overcome any costs associated with meiosis. Surely, parsimony supports a single explanation for the maintenance of sex in both diploid and haploid species rather than hypotheses exclusive to either group.

ROBERT L. DUNBRACK

Department of Biology,

Memorial University of Newfoundland,

St. John's, Newfoundland,

A1B $3 \times 9$ Canada

KirkPatrick AND Jenkins RePly-We showed ${ }^{1}$ that the process of genetic segregation can generate an evolutionary advantage to sexual reproduction that may help explain its prevalence over asexual reproduction. Hedrick and Whittam point out that the segregation advantage we identified will be almost as effective in a population in which most individuals are asexual and only a small fraction are sexual. Such a facultatively sexual population would have the best of both worlds: most of the segregation advantage from sexual reproduction and most of the twofold reproductive advantage from asexual reproduction. Species of this type, however, are virtually unknown among the animals ${ }^{5}$, and so this may not be an evolutionary option. (We do not consider automixis (selfing) here because the ubiquitous deleterious effects of inbreeding $^{6}$ probably select against it in most populations.) We have extended Hedrick and Whittam's observation by considering the more common (though still rare) situation of cyclic parthenogenesis, in which the population undergoes a number of strictly asexual generations followed by a single generation of sexual reproduction ${ }^{5}$. The results are similar to Hedrick and Whittam's: an occasional generation of sexual reproduction is sufficient to give most of the segregation advantage and still retain most of the twofold reproductive advantage of asexuality.

Why then are facultative and cyclic parthenogenesis uncommon? We have two possible explanations. First, the segregation effect we identified is only one of the evoluntionary advantages to sex. Others, such as effects from recombination $^{4.5,7}$, may work in conjunction with the segregation effect to give purely sexual reproduction an overall advantage. Second, constraints may preclude facultative and cyclic parthenogensis as evolutionary options for many taxa $a^{4.5}$. Pure apomictic parthenogensis has appeared dozens of times among the animals, but

\section{More on cold fusion}

SIR-Processes involving cosmic-ray muons have been proposed ${ }^{1-3}$ to explain the small rate of fusion neutrons observed ${ }^{4}$ in the cold-fusion experiments. Although fusion neutrons have not been observed in electrochemical cells placed in muon beams ${ }^{5,6}$, the flux from the control experiment has not been measured. Any coldfusion neutrons of amuonic origin would remain unaffected by the muon beam. These muon experiments therefore demonstrate negative results for cold fusion - both muonic and amuonic in deuterium-charged metals. The muon experiments establish that the deuteron concentration in the samples does not reach abnormally high densities because the muons are lost to the more electropositive host-metal ions as expected for normal ratios of deuterons to metal-ions (up to two). Under conditions of highdensity non-equilibrium deuteron packing as predicted by some of the cold-fusion experiments ${ }^{7}$ and theories, the muon kinetics are not known, but loss to the host metal should be hindered, as it is known to be sometimes delayed ${ }^{8}$ even in ordinary compounds because of intermediate molecular states.

Known cosmic-ray muon fluxes ${ }^{9.10}$, their facultative and cyclic apomictic reproduction have arisen perhaps only half a dozen times ${ }^{5}$. Thus, for most taxa the evolutionary possibilities may be purely sexual or purely asexual reproduction. In this case, the benefits to sex from the effects of segregation and recombination may be sufficient to maintain sexual reproduction.

Dunbrack reiterates two points made in our paper: that there can be a substantial time lag before the segregation advantage offsets the twofold reproductive advantage of asexual reproduction; and that the segregation advantage will be greatly weakened when much or all of the genome is selected in a haploid state for a substantial part of the life cycle. Dunbrack feels these points are fatal to our hypothesis. We disagree based on the arguments presented in our paper, and re-emphasize that there need not be only one evolutionary advantage to sex - the segregation effect can be augmented by others.

\section{MARK KIRKPATRICK} CHERYL D. JENKINS

\section{Department of Zoology,}

\section{University of Texas,}

\section{Austin, Texas 78701, USA}

1. Kirkpatrick, M. \& Jenkins, C.D. Nature 339, 300 (1989).

2. Marshall, D.R. \&Weir, B.S. Heredity 42, 159-172 (1979).

3. Jackson, J.B.C. et al. Population Biology and Evolution of Clonal Organisms (Yale University Press, 1985).

Maynard Smith, J. The Evolution of Sex (Cambridge University Press, 1978).

5. Bell, G. The Masterpiece of Nature: The Evolution and Genetics of Sexuality (University of California Press Berkeley, 1982).

6. Charlesworth, D. \& Charlesworth, B. A. Rev. Ecol. Syst. 18, 237-268 (1987)

7. Michod, R.E. \& Levin, B.R. The Evolution of Sex: An Exam ination of Current ideas (Sinauer, Sunderland, 1988).

stopping rates ${ }^{11}$, and established muoncatalysed-fusion parameters $\mathrm{s}^{12.13}$ indicate that exposure of a large enough volume of liquid deuterium (about 1 litre) to cosmicray muons should also yield a low-level neutron count of about $10^{-2} \mathrm{~s}^{-1}$. There is a possibility of using these fusions for fissilefuel breeding. Although the rate would be extremely slow, the muons would be free and the muon-production cost factor could be dropped from the efficiency calculations for muon-catalysed fusion.

\section{Physics Department,}

LaLI ChatterjeE

\section{Jadavpur University,}

Calcutta 32,

\section{India}

\footnotetext{
1. McCevoy, A.J, \& O'Sullivan, C.T.D. Nature 338, 711 (1989).

2. Chatterjee, L. Preprint JU HEP/89/2, Jadavpur Univ. (1989)

3. Chateriee L. Proc ICENES 1989 (in the press) 4. Jones, S.E. et al. Nature 228, 737-740 (1989)

5. Nagamine, K. Proc. Conf. Muon-Catalysed Fusion (Oxford, 1989) (in the press)

6. Davies, J. Proc. Conf. Muon-Catalysed Fusion (Oxford, 1989) (in the press)

Fleischmann, M. \& Pons, S. J. electroanalyt. Chem. 261 , 301-308 (1989); and erratum, 263. 187-188 (1989).

8. Gerstein, S.S. \& Ponomaryev, L. in Muon Physics Vol. 3 (eds Hughes, V.W. \& Wu, C.S.) (Academic, New York. 1978)

9. Hayakawa, S. in Cosmic Ray Physics Vol. 22 (ed. Marshak R.E.) (Wiley Interscience, New York, 1969)

10. YashPal \& Peters, B. Nat. fys. med. Dam. Vit. S. 33 (1964)

11. Wightman, A.S. Phys. Rev. 77, 521 (1950)

12. Jones, S.E. Nature 321, 127-133 (1986)

13. Chatterjee, L. Phys. Lett. A137, 4-6 (1989).
} 